\title{
Granular collapse in a fluid: Role of the initial volume fraction
}

\author{
L. Rondon, ${ }^{\text {a) }}$ O. Pouliquen, ${ }^{\text {b) }}$ and P. Aussillous ${ }^{\text {c) }}$ \\ Laboratoire IUSTI, UMR 6595 CNRS, Aix Marseille University, 5 rue Enrico Fermi, \\ 13465 Marseille Cedex 13, France
}

(Received 24 December 2010; accepted 5 May 2011; published online 1 July 2011)

\begin{abstract}
The collapse of a granular column in a viscous liquid is experimentally investigated. The morphology of the deposits is shown to be mainly controlled by the initial volume fraction of the granular mass and not by the aspect ratio of the column, an observation which differs from dry granular collapse. Two different regimes are identified corresponding to initially loose and dense packings. Loose packings give rise to thin and long deposits, the dynamics being fast. A positive liquid pressure is measured below the column. For dense packings, the runout distance is twice less, the flow is slow, and a negative pore pressure is measured during the flow. These observations suggest that the dynamics of the granular collapse in a fluid is strongly affected by the dilatancy or contractancy behavior of the granular medium. (C) 2011 American Institute of Physics.
\end{abstract}

[doi:10.1063/1.3594200]

\section{INTRODUCTION}

In nature, instabilities of slopes or cliffs are dramatic events involving the sudden release of a large mass of soil. The flowing mass is mainly composed of a granular material surrounded by an interstitial fluid, which can be gas for aerial landslides, water for submarine flows, and mud for debris flows. Understanding the dynamics of the flow and predicting how fast and how far the mass may travel is important for risks assessment. However, the prediction of catastrophic events still represents a challenge, one difficulty being our incomplete understanding of the dynamics of granular flows. ${ }^{1}$ Small scale laboratory experiments are usually unable to properly capture the dynamics of geophysical events. However, they can be useful to precisely study physical mechanisms, which may play a role in real flows. ${ }^{2-5}$ The study presented in this paper is carried out in this spirit. The goal is to explore the role of the coupling between the grains and the interstitial fluid during the collapse of a granular mass fully immersed in a liquid.

The collapse of a granular column, which mimics the collapse of a cliff, has been extensively studied in the case of dry granular material, when the interstitial fluid plays no role. ${ }^{6,7}$ The first experiments on granular collapse have been carried out in an axisymmetric geometry. A cylinder standing vertically on a plane and initially filled with grains is suddenly lift up. The granular mass rapidly spreads on the plane and ultimately stops, leaving a deposit with a well defined and reproducible morphology. A major observation is that this complex transient flow leads to relatively simple scaling laws: the morphology of the final deposit is mainly controlled by the initial aspect ratio of the column, almost independently of the material properties. Power laws have been observed ${ }^{6-8}$ relating the final height and the final length to the initial aspect ratio. The same problem has been also investigated in a two dimensional geometry, the so-called

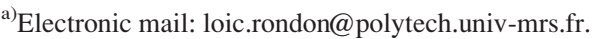

${ }^{\mathrm{b})}$ Electronic mail: olivier.pouliquen@univ-provence.fr.

${ }^{\mathrm{c})}$ Electronic mail: pascale.aussillous@polytech.univ-mrs.fr.
}

dam-break problem. ${ }^{8-13}$ In this case the granular material is initially confined by a wall, which is suddenly removed. The observed scaling characterizing the deposit morphology is different from the axisymmetric case but the aspect ratio remains the relevant parameter, as long as the channel is wide enough to prevent additional frictional effects on the side walls. ${ }^{9}$ The granular collapse has been also studied using numerical simulations based on discrete element methods, allowing a precise study of the internal structure of the flow. ${ }^{13-16}$ From a theoretical point of view, the spreading has been described using depth averaged equations. ${ }^{17-19}$ Although the initial stage of the flow does not fulfill the gentle slope approximation dictated by the depth averaged approach, this approach succeeds in predicting the scaling observed for aspect ratio less than one. However, describing larger aspect ratio and capturing the initial stage of the collapse, when the grains experience a rapid change of direction from vertical to horizontal, remain an open challenge. ${ }^{15}$

The case of the collapse in presence of an interstitial fluid has been less studied. Roche et al. ${ }^{5,20}$ have investigated the role of air in the propagation of the surge in the dam break configuration. They have shown that air can strongly enhance the mobility of the mass when the particles are fine enough and when the initial pile is fluidized by an injection of air. The problem of the granular collapse in a liquid, which is of importance for submarine landslides, has to our knowledge attracted less attention. A lot of work has been devoted to turbidity currents, addressing the problem of the spreading of a low concentrated suspension in a clear fluid. ${ }^{21}$ However, these flows are far from the dense granular regime observed in collapse, when particles are initially in contact. Thompson and Huppert ${ }^{22}$ in their paper rapidly discuss an experiment of a granular collapse in water. They show that the liquid dramatically changes the way the column collapses compared to the dry case. Bonnet et $a .^{23}$ have also studied submarine avalanches in a confined geometry. A pile of grains confined on the side of a square box by an horizontal counter current of water is suddenly released by stopping the counter current. A major result of their study is that the 
destabilization of the pile strongly depends on the initial volume fraction of the packing. For dense packings the granular flow is localized at the free surface of the pile, whereas for loose packings the destabilization occurs in the bulk of the material and take the shape of a lens.

The role of the initial density in the motion a granular mass has been shown in the striking experiment of Iverson et $a l .{ }^{24}$ Using a large scale facility, they have shown that a soil prepared in a loose state on a slope and submitted to a rainfall flows rapidly like a liquid when it breaks, whereas a dense soil only slowly creeps. The underlying mechanism is related to the dilatant or contractant character of the granular material and has been called "the pore pressure feedback." ${ }^{24,25}$ A compaction or a dilatation of the granular material during the initial deformation induces a relative motion between the grains and the fluid, which in turns induces an additional stress on the granular skeleton. This additional stress can stabilize or destabilize the soil. Recently, a small scale experiment carried out in the viscous regime has given a precise and quantitative analysis of this coupling. ${ }^{26}$ The crucial role of the initial volume fraction has been analyzed by investigating how a thin layer of grains immersed in a liquid starts to flow when it is suddenly inclined from horizontal. The dynamics observed in this experiment can be captured theoretically using the framework of two phase flow equations. ${ }^{27,28}$

The main goal of this paper is to study how the pore pressure feedback mechanism may affect the collapse of an immersed granular column. Our experiment consists in a dam-break configuration in a viscous liquid, in which we control the initial volume fraction of the column. The aim is to investigate how the collapse of a dense column differs from the collapse of a loose column.

The paper is organized as follows. The experimental setup and the procedure are presented in Sec. II. The differences observed between an initially loose and an initially dense packing are first qualitatively described in Sec. III. In Sec. IV, we quantitatively study the morphology of the final deposit. Section V is devoted to the dynamics of the flow and how it changes when changing the initial volume fraction. Section VI is a discussion, where our observations for the immersed case are compared to results from the dry case. Finally, a conclusion is presented in Sec. VII.

\section{EXPERIMENTAL SET UP AND PROCEDURE}

The experimental set-up is presented in Fig. 1. It consists in a perspex box $70 \mathrm{~cm}$ long, $15 \mathrm{~cm}$ wide, and $15 \mathrm{~cm}$ high, full of liquid. The bottom surface of the box is made rough by gluing a layer of particles on it. A vertical wall can be positioned at one end of the tank to delimit a reservoir for the granular material. This wall is maintained by two slots made on the side walls of the tank and can be removed rapidly to simulate a dam-break. Three different positions are possible for the retaining wall corresponding to three different reservoir widths $L_{i}=2, L_{i}=4$, and $6 \mathrm{~cm}$. The particles used are glass beads of density $\rho_{s}=2500 \mathrm{~kg} \mathrm{~m}^{-3}$ and $d=225 \mu \mathrm{m}$ in mean diameter. The liquid is a mixture of water and Ucon oil 75H90000 from Dow, a viscous newtonian liquid soluble in water. Two mixtures are used, the first

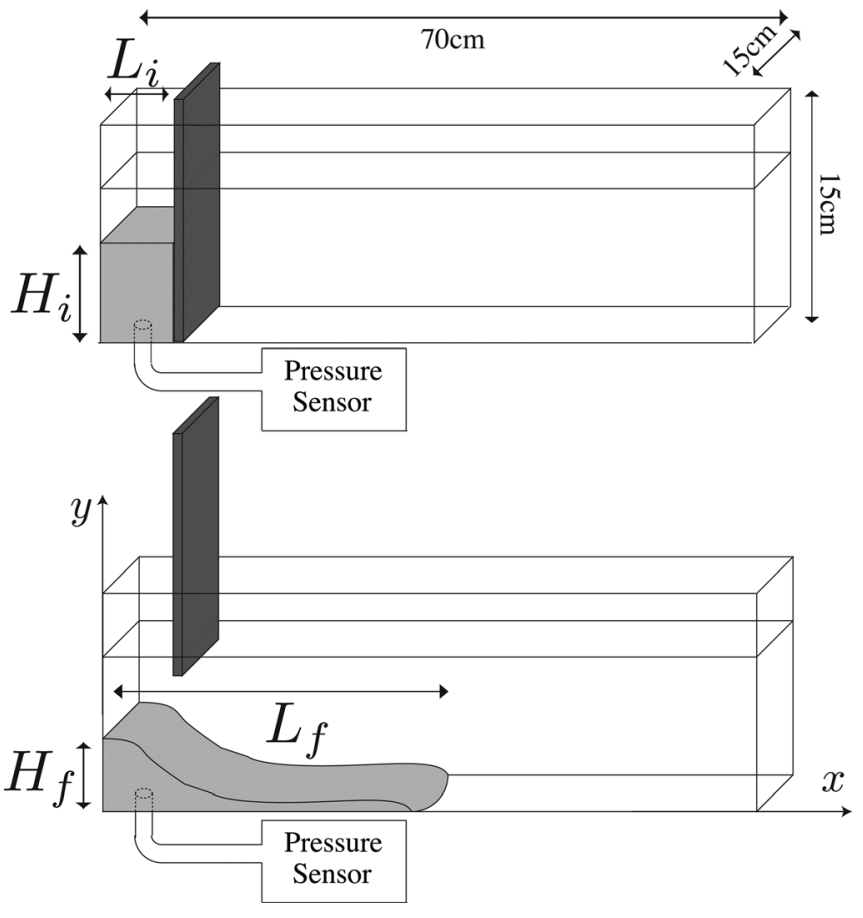

FIG. 1. Experimental set-up.

one made of $87 \%$ of water and $13 \%$ of Ucon with a viscosity $\eta=12$ and the second made of $83 \%$ of water and $17 \%$ of Ucon with $\eta=23 \mathrm{cP}$. In order to measure the pore pressure under the pile, a $3 \mathrm{~mm}$ hole is drilled at $2 \mathrm{~cm}$ from the end of the tank. This hole is closed with a $40 \mu \mathrm{m}$ mesh, preventing the particles to go through. The hole is connected by a tube to one end of a differential piezo electric pressure sensor, Honeywell DC005NDC4, the second end being at the atmospheric pressure. The pressure signal thus gives the pressure of the liquid under the pile.

The experimental procedure is the following. The tank being full of liquid, the retaining wall is placed at the desired position. Glass beads already mixed with liquid are then gently poured in the reservoir delimited by the wall. The liquid level is then adjusted on both sides of the gate by adding or removing liquid with a syringe. The grains are then put in suspension in the reservoir using a small propeller driven by a drill motor. The propeller is moved randomly in order to obtain a uniform particle distribution. Once the propeller is stopped, we wait until all the particles have sedimented. A clear signature of the end of the sedimentation process is given by the pore pressure signal, which continuously decreases during the sedimentation and stops decreasing when the static pile is formed. Using this procedure, we then obtain a packing with a flat free surface, which is in the loosest state we can create. The solid volume fraction $\phi_{s}$ in this loose state just after sedimentation is found ${ }^{29}$ to be equal to $\phi_{s}=0.55 \pm 0.005$. We have checked that this value is reproducible and independent of the amount of grains and of the size of the reservoir. To create denser packings, we then gently kick on the tank. Measuring the heights of the column $H_{s}$ and $H_{i}$ before and after compaction gives an estimate of the mean volume fraction $\phi_{i}$ of the column after compaction: $\phi_{i}=\phi_{s} \frac{H_{s}}{H_{i}}$. The range of volume fraction reached in our set up is $0.55 \lesssim \phi_{i} \lesssim 0.62$. The error bar on our estimate of the 
TABLE I. List of the experiments. Experiments are sorted by increasing aspect ratio $\mathcal{A}=\frac{H_{i}}{L_{i}}$.

\begin{tabular}{cccccccccc}
\hline \hline & $\begin{array}{c}L_{i} \\
(\mathrm{~cm})\end{array}$ & $\begin{array}{c}\eta \\
(\mathrm{cP})\end{array}$ & $\mathcal{A}$ & $\begin{array}{c}H_{i} \\
(\mathrm{~cm})\end{array}$ & $\begin{array}{c}L_{i} \\
(\mathrm{~cm})\end{array}$ & $\begin{array}{c}\eta \\
(\mathrm{cP})\end{array}$ & $\mathcal{A}$ & $\begin{array}{c}H_{i} \\
(\mathrm{~cm})\end{array}$ \\
\hline 1 & 6 & 12 & 0.37 & 2.2 & 7 & 6 & 12 & 1.33 & 7.97 \\
2 & 4 & 23 & 0.52 & 2.08 & 8 & 4 & 23 & 1.51 & 6.06 \\
3 & 6 & 12 & 0.74 & 4.42 & 9 & 4 & 23 & 1.97 & 7.87 \\
4 & 4 & 23 & 0.78 & 3.12 & 10 & 2 & 12 & 1.98 & 3.95 \\
5 & 4 & 23 & 0.99 & 3.95 & 11 & 2 & 12 & 3.05 & 6.09 \\
6 & 6 & 12 & 1.03 & 6.21 & 12 & 2 & 12 & 4.55 & 9.10 \\
\hline \hline
\end{tabular}

volume fraction depends on the height of the column and is \pm 0.013 for $H_{i}=2 \mathrm{~cm}$ and \pm 0.003 for $H_{i}=10 \mathrm{~cm}$. Note that only the depth averaged volume fraction is measured and that variations could exist in the column. However, previous works on tap induced compaction of granular matter suggest that the compaction process is homogeneous. ${ }^{30}$

Once the column is prepared at the desired mean volume fraction $\phi_{i}$, the gate is suddenly removed by hand [Fig. 1(b)]. The column collapses and creates a surge which propagates in the box. No noticeable transverse variation is observed during the flow, indicating that wall effects are negligible and that the flow is essentially two-dimensional. A side view using a video camera at 30 frames per second with a resolution of $1920 \times 1080$ pixels is then sufficient to analyze the flow dynamics. The pore pressure signal under the pile is recorded at $50 \mathrm{~Hz}$ using an acquisition board.

In order to explore the role of the different parameters, we have carried out experiments changing the height $H_{i}$ of the column, the width $L_{i}$ of the column, and the viscosity $\eta$ of the fluid. The different experimental conditions are summarized in Table I. In our set-up, the aspect ratio of the initial column $\mathcal{A}=\frac{H_{i}}{L_{i}}$ is limited to the range $0.33<\mathcal{A}<4.55$. For each condition listed in Table I, the initial volume has been varied in the range $0.55 \lesssim \phi_{i} \lesssim 0.62$.

\section{EVIDENCE OF THE ROLE OF THE INITIAL VOLUME FRACTION}

Before studying systematically the role of the different parameters in the spreading of the granular mass, we first present evidences that the initial volume fraction of the pile plays a major role. Figure 2 shows the time evolution of the same granular mass prepared in a dense state $\left(\phi_{i}=0.6\right)$ and in a loose case $\left(\phi_{i}=0.55\right)$. Movies can be found in the online ver-

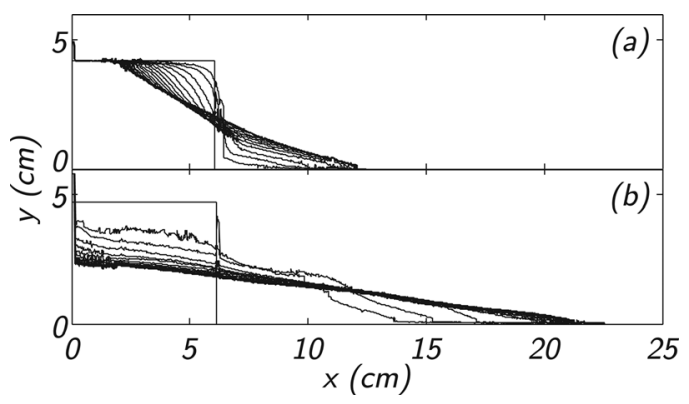

FIG. 2. Collapse of a dense and a loose column containing the same amount of sand for an aspect ratio $\mathcal{A} \simeq 0.67$. (a) $\phi_{i}=0.60, L_{i}=6 \mathrm{~cm}, H_{i}=4.2$ $\mathrm{cm}, 3 \mathrm{~s}$ between profiles; (b) $\phi_{i}=0.55, L_{i}=6 \mathrm{~cm}, H_{i}=4.8 \mathrm{~cm}, 0.66 \mathrm{~s}$ between profiles. sion. When opening the door, the initially dense column does not immediately collapse. Grains only move at the right upper corner, where a slow erosion takes place, leading to a very abrupt profile with a round right corner and a small deposit at the feet of the column. When time goes on, the eroded corner becomes larger and larger and the profile is less and less steep. Eventually, the deposit exhibits a trapezoidal shape. The left part has not moved and presents a plateau at the same thickness as the initial column. The right part is roughly triangular. During the collapse of these dense columns, failures occasionally are observed, where part of the right corner detaches as a block and falls at the feet of the column.

The dynamics of the loose case is different [Fig. 2(b)]. First, contrary to the dense case, the whole column is mobilized immediately after the opening: grains at the left corner of the column also move down. Secondly, the column spreads further than in the dense case. The runout of the granular mass is about twice longer when starting from a loose state than from a dense state. The final shape of the deposit is then much more elongated. Thirdly, the motion is more rapid in the loose case than in the dense case. This is shown by comparing the spacing between the successive profiles plotted in Fig. 2, knowing that they are plotted every $0.66 \mathrm{~s}$ in Fig. 2(b) and every $3 \mathrm{~s}$ in Fig. 2(a). The rapid dynamics of the loose case are also clearly observed in Fig. 3(a) where the position of the surge front is plotted versus time for the two cases. The spreading of the initially loose column is very rapid at the beginning and slows down before it stops, whereas the spreading of the dense column is much slower.

A last but important difference between the dense and loose collapse is observed on the pore pressure signal measured below the initial column. Figure 3(b) shows how the pressure of the water under the pile varies with time during the collapse. The hydrostatic pressure has been subtracted to the signal, such that the reference pressure before the opening of the gate is 0 . During the collapse of the dense column, a negative pressure is observed, whereas a positive pore pressure is observed for the collapse of the loose column. This is similar to what is observed during the initial motion of a thin layer of grains, and suggests that fluid is sucked into the granular skeleton when the column is initially dense,
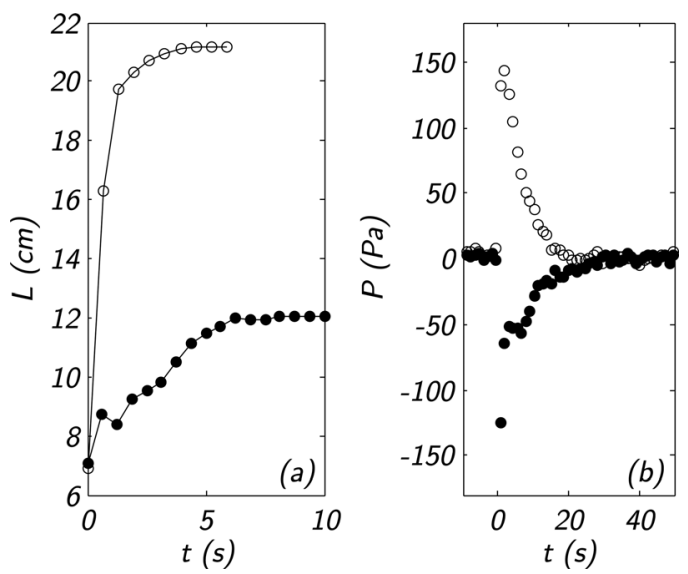

FIG. 3. (a) Time evolution of the front position for the dense (•) and loose (o) cases of Fig. 2; (b) corresponding pore pressure evolution below the column (enhanced online). [URL:http://dx.doi.org/10.1063/1.3594200.1] 

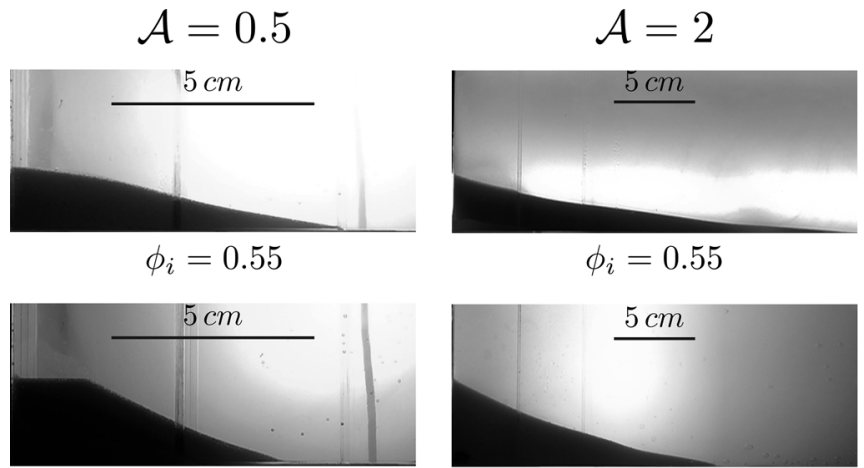

$\phi_{i}=0.58$

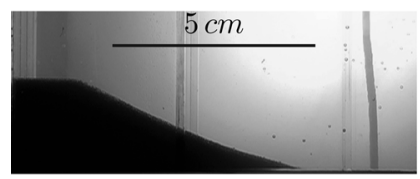

$\phi_{i}=0.61$

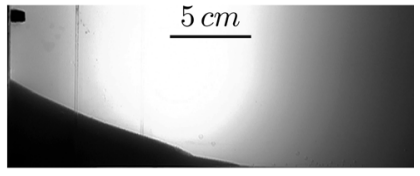

$\phi_{i}=0.61$

FIG. 4. Deposit observed for $L_{i}=4 \mathrm{~cm}$ and $H_{i}=2 \mathrm{~cm}, \mathcal{A}=0.5$ for the left column and $H_{i}=8 \mathrm{~cm}, \mathcal{A}=2$ for the right column.

whereas fluid is ejected out of the granular skeleton in the loose case.

These preliminary observations then strongly suggest that the initial volume fraction is a crucial parameter in the study of the granular collapse in a fluid. In Sec. IV, we analyze how the final morphology and the flow dynamics vary with the initial aspect ratio and the initial volume fraction.

\section{MORPHOLOGY OF THE DEPOSIT}

Examples of deposits observed at the end of the collapse are presented in Fig. 4. Pictures correspond to two different aspect ratios, $\mathcal{A}=0.5$ and $\mathcal{A}=2$, and different volume fraction. Two different morphologies are observed. At low aspect ratio and high volume fraction, the deposit looks like a trapezoid, with a flat surface on the left side reminiscent of the initial packing and a triangle on the right side resulting from the avalanche of the right corner. At higher aspect ratio or/and lower initial volume fraction, the deposit no longer presents a flat surface but is more or less triangular, the final height being less than the initial height.

The transition between a trapezoidal and a triangular shape is presented in Fig. 5. For different aspect ratios and initial volume fractions we indicate whether the deposit presents a plateau $(\square)$ or not $(\triangle)$. The figure shows that for dense packing $\phi_{i} \gtrsim 0.58$, the trapezoid is observed when $\mathcal{A}<0.8$. This value is closed to the one observed in the collapse of dry columns. ${ }^{8}$ This critical aspect ratio is no longer constant for $\phi_{i} \lesssim 0.58$ and becomes lower and lower when $\phi$ decreases.

In the sequel, we focus on the morphology of the deposits presenting no plateau observed for high enough aspect ratio. In order to compare their shape, we have plotted in Fig. 6 the profiles normalized by the final height $h / H_{f}$ as a function of the horizontal distance normalized by the final length $x / L_{f}$. This figure shows that the profiles obtained for different experimental conditions have roughly the same shape independently of $\phi_{i}, \eta, H_{i}$, and $L_{i}$. The shape is close to a triangle but is in averaged slightly concave. The concavity can be quanti-

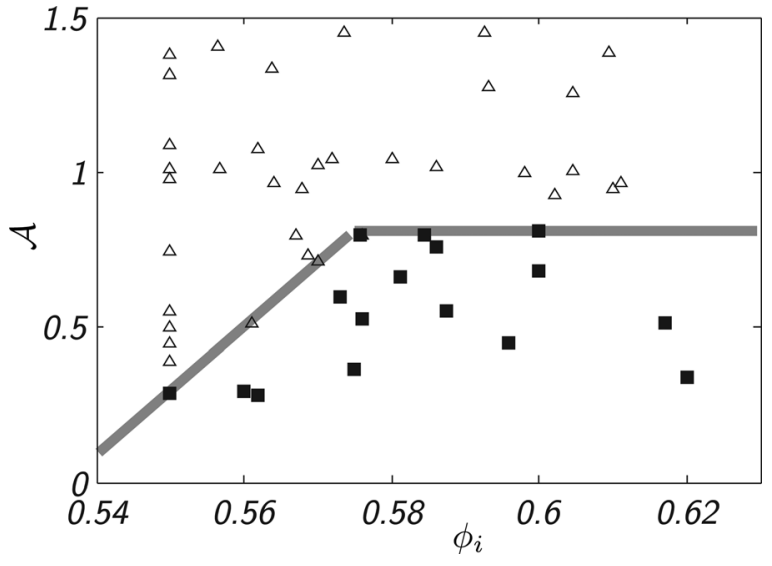

FIG. 5. The two deposits morphologies observed (triangle $\triangle$, trapezium in the $\left(\mathcal{A}, \phi_{i}\right)$ plane.

fied by plotting how the product $H_{f} L_{f}$ varies with $H_{i} L_{i}$ (Fig. $7)$. For a perfect triangle, the mass conservation implies that $H_{f} L_{f}=2 H_{i} L_{i}$. Our data collapse around a straight line but with a slope 2.55 slightly higher than 2 , indicating that in average the deposit profiles present a small concavity.

All the profiles being roughly self similar with a shape close to a triangle, the analysis of the morphology can be reduced to the measurement of the apparent slope of the deposit $H_{f} / L_{f}$ and its variation with the aspect ratio $\mathcal{A}$ and the initial volume fraction $\phi_{i}$. Figure 8(a) shows how $H_{f} / L_{f}$ varies with $\mathcal{A}$ for two initial volume fractions corresponding to a dense case $\left(\phi_{i}=0.6\right)$ and a loose case $\left(\phi_{i}=0.55\right)$. The deposit resulting from the collapse of the dense packing exhibits a slight decrease of the apparent slope with $\mathcal{A}$ for $\mathcal{A}<2$ and remains constant for larger $\mathcal{A}$. The ratio $H_{f} / L_{f}$ varies between 0.4 and 0.35 , which corresponds to an apparent angle of the deposit between $22^{\circ}$ and $19^{\circ}$. In the loose case, the slope is much lower, varying between 0.15 and 0.2 corresponding to an angle between $8.5^{\circ}$ and $11^{\circ}$. This observation contrasts with what is observed in the dry case, ${ }^{6-9,22}$ for which the apparent slope depends on the aspect ratio [Fig. 8(a)], a point we will discuss in more detail in Sec. VI. The morphology of the granular collapse in a viscous fluid is thus mainly controlled by the initial volume fraction $\phi_{i}$ and not by the initial aspect ratio $\mathcal{A}$, at least for the moderate aspect ratio we have been able to create in our setup. We can

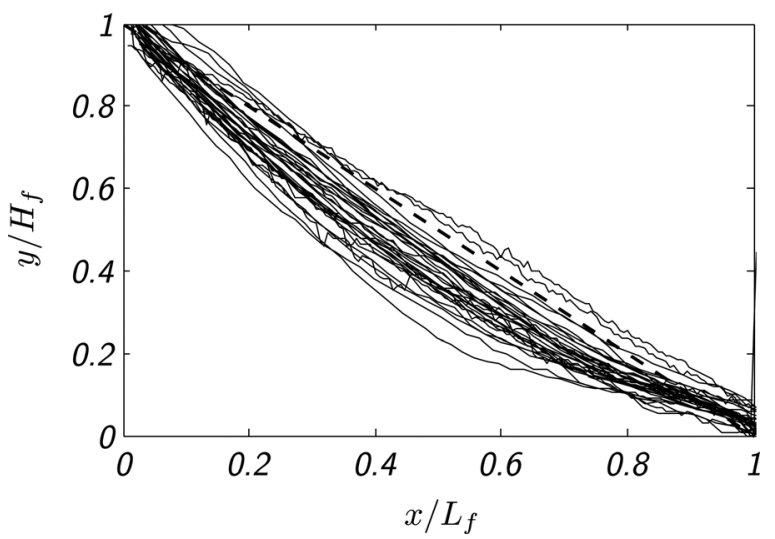

FIG. 6. Dimensionless shape of the deposit without flat part. The dashed line represents the curve $\frac{y}{H_{f}}=\frac{x}{L_{f}}$. 


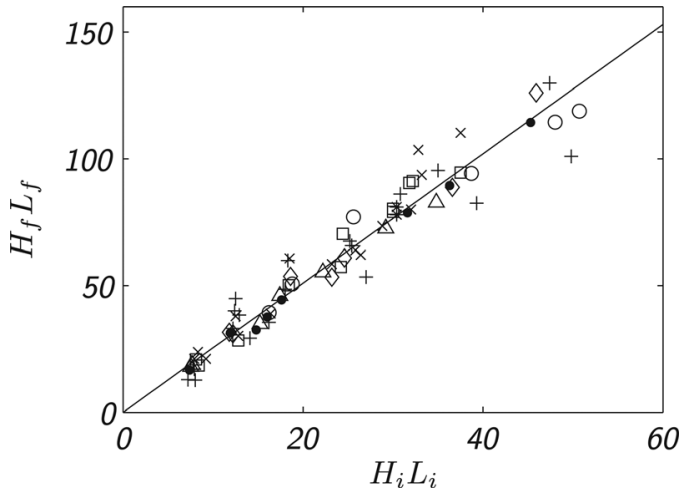

FIG. 7. $H_{f} L_{f}$ as a function of $H_{i} L_{i}$ for triangular deposit. $\phi_{i}=0.55(+)$, $0.56(\times), 0.57(\square), 0.58(\triangle), 0.59(\diamond), 0.60(\bullet), 0.61(\circ)$. The solid line corresponds to a slope 2.55 .

then plot the apparent slope as a function of the initial volume fraction $\phi_{i}$ for all our runs independently of the aspect ratio [Fig. 8(b)]. Although the data are scattered, we can again distinguish between a loose and a dense behavior. For a low initial volume fraction $\phi_{i}=0.55$ the final slope is as small as $0.15\left(8.5^{\circ}\right)$, which corresponds to very elongated deposits. The slope then increases roughly linearly when increasing $\phi_{i}$ and reaches values about $0.37\left(20^{\circ}\right)$ for $\phi \gtrsim .59$, which corresponds approximately to the angle of repose of the material. The value 0.37 for the slope of dense packings is consistent with the transition between trapezoid and triangle deposits, observed for a critical aspect ratio $\mathcal{A} \simeq 0.8$ (Fig. 5). At the transition one has $H_{i}=H_{f}$; considering that the slope of the triangle is equal to the angle of repose $H_{f} / L_{f} \simeq 0.37$ and that $H_{f} L_{f}=2 H_{i} L_{i}$ from mass conservation, one predicts that the critical aspect ratio for the transition is given by $H_{i} / L_{i}=2 H_{f} / L_{f} \simeq 0.74$.

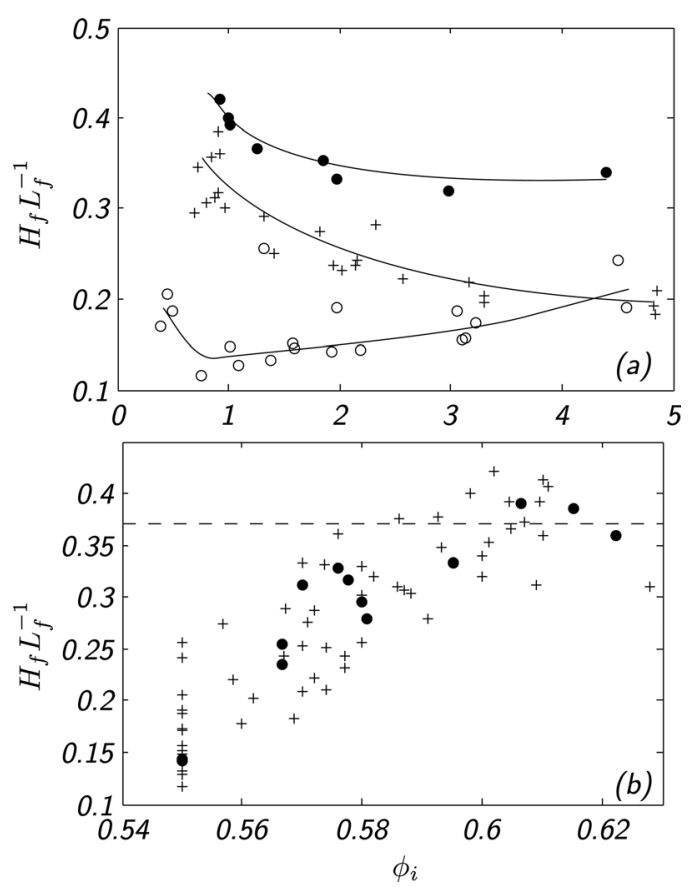

FIG. 8. (a) Apparent slope $\frac{H_{f}}{L_{f}}$ as a function of $\mathcal{A}$ for $\phi_{i}=0.55(\mathrm{o})$, $\phi_{i}=0.60(\bullet)$, and dry $^{6}(+)$. The lines are guides for the eyes. (b) Apparent slope $\frac{H_{f}}{L}$ as a function of $\phi_{i}$ for triangular deposit for all runs $(+)$, except $\mathcal{A}=1.97(\bullet)$, set n 9 on Table I.

\section{DYNAMICS OF THE SPREADING}

We have seen that the final morphology of the deposit is mainly controlled by the initial volume fraction $\phi_{i}$ of the packing. In this section we analyze the influence of $\phi_{i}$ on the typical time it takes before the flow stops and on the level of pore pressure measured below the column.

In order to investigate the dynamics, we compute for each run the time $t_{95}$ defined as the time it takes for the surge to travel a distance equal to $95 \%$ of its final position. Determining $t_{95}$ is more precise than trying to measure the time it takes for the flow to stop. The Fig. 9(a) shows how the time $t_{95}$ varies with the initial volume fraction for a fixed initial geometry $H_{i}$ and $L_{i}$. At low volume fraction, the spreading time is about few seconds and is relatively constant, but it dramatically increases when $\phi_{i}$ goes above 0.58 , increasing up to $40 \mathrm{~s}$ for $\phi_{i}=0.62$. The collapse of a dense column can then take 10 times longer than the collapse of a loose column. In order to compare the time measurements obtained for different initial geometries and different fluid viscosities, we have tried different scaling to nondimensionalise the time scale. The best collapse of our data is obtained when we choose a characteristic time $t_{0}$, which scales like ${ }^{26}$ $t_{0}=\eta / \Delta \rho g H_{i}$. This typical time is obtained by writing a balance between the gravitational stress created by the weight of the column $\Delta \rho g H_{i}$ and a typical viscous stress $\eta \dot{\gamma}$, where $\dot{\gamma}$ is the shear rate. The typical shear rate is $\dot{\gamma} \propto \frac{\Delta \rho g H_{i}}{\eta}$. The time scale $t_{0}$ is found by saying that it is the time it takes for a deformation of order one to occur, which means $t_{0}=\dot{\gamma}^{-1}$.
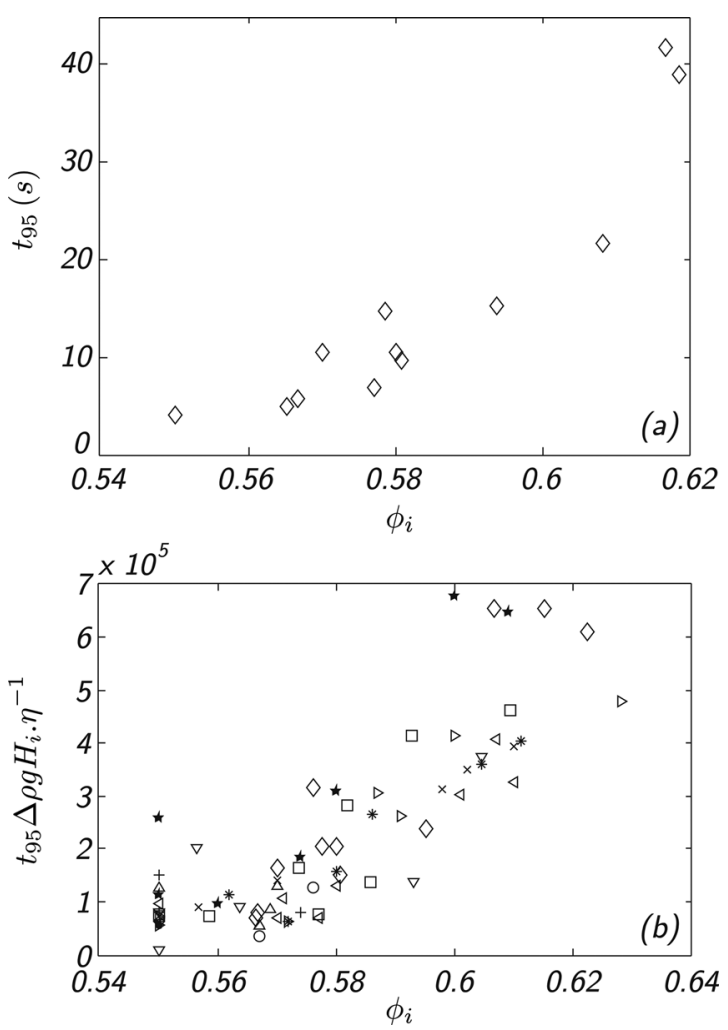

FIG. 9. (a) $t_{95}$ in second as a function of $\phi_{i}$, (b) $t_{95} / t_{0}$ as a function of $\phi_{i}$. Runs using two different fluids are presented: $\eta=12 \mathrm{cP}, \mathcal{A}=0.37(\bullet)$, $\mathcal{A}=0.74(\triangle), \quad \mathcal{A}=1.03(*), \quad \mathcal{A}=1.33(\nabla), \quad \mathcal{A}=1.98(\triangleleft), \quad \mathcal{A}=3.05(\triangleright)$, $\mathcal{A}=4.55(\star) ; \quad \eta=23 \quad \mathrm{cP}, \quad \mathcal{A}=0.52(+), \quad \mathcal{A}=0.78(\circ), \quad \mathcal{A}=0.99(\times)$, $\mathcal{A}=1.51(\square), \mathcal{A}=1.97(\diamond)$. 
Using $t_{0}$ as a time scale, a reasonable collapse of all our data is obtained when plotting $t_{95} / t_{0}$ as a function of the initial volume fraction $\phi_{i}$ as shown in Fig. 9. Again, two distinct behaviors are observed depending on the value of $\phi_{i}$. The collapse of a loose packing when $\phi_{i} \lesssim 0.58$ typically last a time equal to $10^{5} \cdot t_{0} / 2$ independent of $\phi_{i}$. The collapse of a dense packing strongly depends on the initial compaction and the time increases from $5.10^{4} t_{0}$ up to $4.10^{5} t_{0}$ when $\phi_{i}$ increases from 0.58 to 0.62 . We can then conclude that the time of a collapse is also mainly controlled by the initial volume fraction.

A last signature of the difference between the collapse of loose and dense samples can be found in the pore pressure measurements below the column. For each run, we can extract from the time record of the pressure [Fig. 3(b)] the extreme value reached by the signal during the collapse. It can be either positive or negative as already discussed in Sec. III. Figure 10 shows how these extrema vary when changing the initial volume fraction $\phi_{i}$. In this graph, the pressure is made dimensionless using the characteristic pressure $\Delta \rho g H_{i}$. First, we observed that a transition occurs around $\phi_{i} \simeq 0.58$, separating a region where positive pressure is measured and a region where the pore pressure is negative. The positive pressure measured in loose packings means that a partial fluidization occurs, where part of the weight of the grains is carried by the fluid. Notice that the maximum pressure we have measured in Fig. 10, corresponds to $20 \%$ of the total mass of the grains (a total fluidization would give a dimensionless pressure equal to one in Fig. 10). The level of negative pressure measured in the dense cases is of the same order of magnitude. It corresponds to fluid sucked into the granular packing, leading to an additional confining stress on the packing. The pressure measurements are strong evidence that the difference between dense and loose packings is related to the coupling between the deformation of the granular skeleton and the fluid.

\section{COMPARISON WITH DRY GRANULAR COLLAPSE}

We have shown that the collapse of a granular column in a viscous fluid is mainly controlled by the initial volume fraction and not by the aspect ratio of the column. This result contrasts with the collapse of dry granular media previously studied. ${ }^{8,11}$ In this case, the aspect ratio of the column plays a major role. In order to compare more quantitatively the vis-

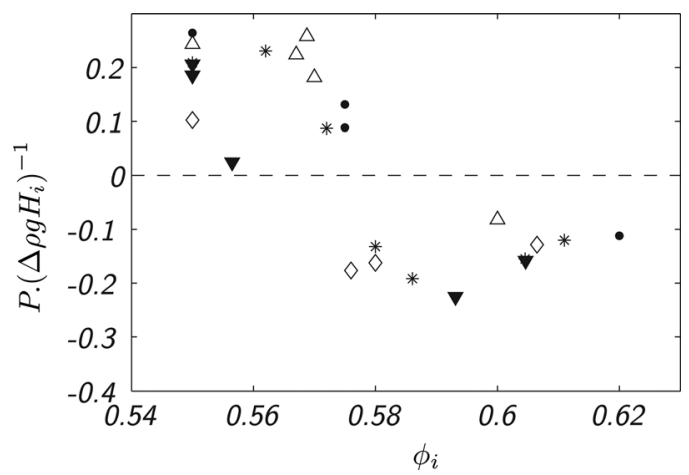

FIG. 10. Extremum dimensionless pore pressure as a function of $\phi_{i}$; $\mathcal{A}=1.97$ and $\eta=12 \mathrm{cP}(\bullet), \mathcal{A}=0.37$ and $\eta=24 \mathrm{cP}(\diamond), \mathcal{A}=0.74$ and $\eta=24 \mathrm{cP}(\boldsymbol{\nabla}), \mathcal{A}=1.03$ and $\eta=24 \mathrm{cP}(\triangle), \mathcal{A}=1.33$ and $\eta=24 \mathrm{cP}(*)$ cous collapse and the dry collapse, we have plotted in Figs. 11 the final height rescaled by the initial length $H_{f} / L_{i}$ and the final length rescaled by the initial length $L_{f} / L_{i}$ as a function of the aspect ratio for the dry, loose, and dense cases. These are the standard plots found in the literature. In Fig. 11(a) the data from the dry and dense immersed cases coincide for $\mathcal{A}<0.7$. In this range the data correspond to trapezoidal deposits for which the final height is equal to the initial one, leading to the trivial scaling $H_{f} / L_{i}=\mathcal{A}$. For larger aspect ratio, the data from the dry case deviate from the dense case, the final heights being smaller. A similar trend is observed in Fig. 11(b), although less pronounced. However, the differences in behaviors between the different cases are not easy to analyze using Figs. 11. The raison is that the differences are masked by a general trend which can be explained by geometrical arguments. If one assumes that the final deposits are triangle with slope $\alpha$, one get $H_{f} / L_{f} \simeq \tan \alpha$. Mass conservation implies $H_{f} L_{f} \simeq 2 H_{i} L_{i}$. One then get the following scaling laws: $H_{f} / L_{i}=\sqrt{2 \tan \alpha \mathcal{A}}$ and $L_{f} / L_{i}=\sqrt{2 \mathcal{A} / \tan \alpha}$. The variation with the square root of $\mathcal{A}$ predicts a slope $1 / 2$ in the log-log plot of Figs. 11. We observe that this slope captures the main tendency in both plots. The difference in mobility in then hidden in the deviation from this simple scaling. This suggests that a better way to characterize the mobility is to study how the apparent slope of the deposit varies with the parameters. This corresponds to Fig. 8(a), where $H_{f} / L_{f}$ is plotted for both our data in immersed conditions and data for dry collapse (from Lajeunesse et $a l .{ }^{8}$ ). The slope observed in dry deposit (crosses) decreases with $\mathcal{A}$ from 0.37 for $\mathcal{A}=0.7$ to 0.19 for $\mathcal{A}=4.9$, whereas the variation is much less for both dense
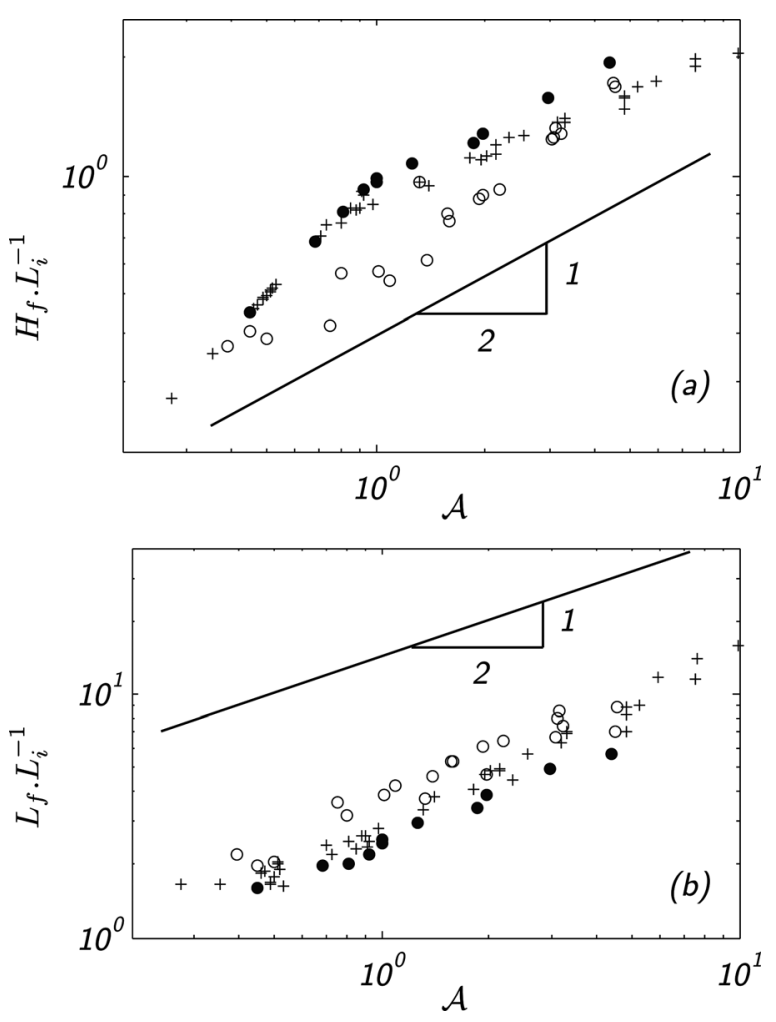

FIG. 11. (a) $\frac{H_{f}}{L_{i}}$ function of $\mathcal{A} ; \phi_{i}=0.55(\circ), \phi_{i}=0.60(\bullet)$, dry ${ }^{6}(+)$. (b) $\frac{L_{f}}{L_{i}}$ function of $\mathcal{A} ; \phi_{i}=0.55(\circ), \phi_{i}=0.60(\bullet)$, $\operatorname{dry}^{6}(+)$. 
and loose immersed cases. In the dry case, inertia is responsible for the enhance of mobility at high aspect ratio. In the viscous regime of interest in our study, inertial effects remain negligible, which could explain why the important parameter controlling the dynamics is the initial volume fraction and not the initial aspect ratio. However, it is worth to mention that the role of the volume fraction on dry granular collapse has not been precisely studied and the preparation of the pile may also play a role (see for example the work by Daerr and Douady ${ }^{31}$ ).

\section{CONCLUSION}

In this paper, we have presented experimental results for the collapse of a granular column in a viscous liquid. We have shown that the shape of the deposit strongly depends on the initial volume fraction of the material and only slightly varies with the initial aspect ratio of the column. Two different behaviors have been identified corresponding to a loose and a dense regime. Loose packings flow rapidly on a time scale proportional to the initial height. They give rise to elongated final deposits with a slope that decreases when decreasing the volume fraction. By contrast, dense packings flow much slower on a time scale that increases when increasing the volume fraction. The final slope of the deposit is in this case roughly constant and equal to the angle of repose of the material. The transition between the two regimes seems to occur around a critical volume fraction equal to 0.58 . The role of the initial volume fraction observed in the viscous collapse can be understood by the pore pressure feedback mechanism proposed by Iverson et $a l^{24,25}$ in the context of landslides. The dense column needs to dilate in order to flow. When it starts to fall, liquid is then sucked into the column, which is then stabilized by the additional viscous drag. By opposition the loose column when it starts flowing expands and ejects liquid, leading to a partial fluidization of the material. Our measurements of pore pressure below the column, which show that positive pressure (resp. negative) develops for loose (resp. dense) columns, are a signature of this process. Going further than this qualitative argument and modeling the dynamics of granular collapse in a liquid remains a challenge, which needs to take into account the coupling between grains and liquid. The approach based on two-phase flow depth averaged equations develope ${ }^{27}$ for the case of uniform avalanches could be a relevant framework.

\section{ACKNOWLEDGMENTS}

We thank Eric Lajeunesse for discussion and for sending us his data. Funding from Agence Nationale de la Recherche (Project RSC ANR-08-BLAN-0048-CSD2) and the IndoFrench Centre for the Promotion of Advanced Research (IFCPAR Project No. 3404-1) is gratefully acknowledged.

${ }^{1}$ R. M. Iverson, "The physics of debris flows," Rev. Geophys. 35, 245, doi:10.1029/97RG00426 (1997).

${ }^{2} \mathrm{O}$. Pouliquen and J. Vallance, "Segregation induced instabilities of granular fronts," Chaos 9, 621 (1999).
${ }^{3}$ R. M. Iverson, M. Logan, and R. P. Denlinger, "Granular avalanches across irregular three-dimensional terrain: II. Experimental tests," J. Geophys. Res. 109, F01015, doi:10.1029/2003JF000084 (2004).

${ }^{4}$ E. Lajeunesse, C. Quantin, P. Allemand, and C. Delacourt, "New insights on the runout of large landslides in the Valles-Marineris Canyons, Mars," J. Geophys. Res. 33, L04403, doi:10.1029/2005GL025168 (2006)

${ }^{5}$ O. Roche, S. Montserrat, Y. Nio, and A. Tamburrino, "Experimental observations of water-like behavior of initially fluidized, dam break granular flows and their relevance for the propagation of ash-rich pyroclastic flows," J. Geophys. Res. 113, B12203, doi:10.1029/2008JB005664 (2008).

${ }^{6} \mathrm{E}$. Lajeunesse, A. Mangeney-Castelnau, and J. P. Vilotte, "Spreading of a granular mass on a horizontal plane," Phys. Fluids 16, 2371 (2004).

${ }^{7}$ G. Lube, H. Huppert, and R. Sparks, "Axisymmetric collapses of granular columns," J. Fluid Mech. 508, 175 (2004).

${ }^{8}$ E. Lajeunesse, J. Monnier, and G. Homsy, "Granular slumping on a horizontal surface," Phys. Fluids 17, 103302 (2005).

${ }^{9}$ N. J. Balmforth and R. R. Kerswell, "Granular collapse in two dimensions," J. Fluid Mech. 538, 399 (2005).

${ }^{10}$ S. Siavoshi and A. Kudrolli, "Failure of a granular step," Phys. Rev. E 71, 051302 (2005).

${ }^{11}$ G. Lube, H. Huppert, R. Sparks, and A. Freundt, "Collapses of two-dimensional granular columns," Phys. Rev. E 72, 041301 (2005).

${ }^{12}$ G. Lube, H. Huppert, R. Sparks and A. Freundt, "Static and flowing regions in granular collapses down channels," Phys. Fluids 19, 043301 (2007).

${ }^{13}$ L. Lacaze, J. C. Phillips, and R. R. Kerswell, "Planar collapse of a granular column: Experiments and discrete element simulations," Phys. Fluids 20, 063302 (2008).

${ }^{14}$ R. Zenit, "Computer simulations of the collapse of a granular column," Phys. Fluids 17, 031703 (2005).

${ }^{15}$ L. Lacaze and R. R. Kerswell, "Axisymmetric granular collapse: A transient 3D flow test of viscoplasticity," Phys. Rev. Lett. 102, 108305 (2009).

${ }^{16} \mathrm{~L}$. Staron and E. J. Hinch, "The spreading of a granular mass: Role of grain properties and initial conditions," Granular Matter 9, 205 (2007).

${ }^{17}$ R. R. Kerswell, "Dam break with Coulomb friction: A model for granular slumping?," Phys. Fluids 17, 057101 (2005).

${ }^{18}$ A. Mangeney-Castelnau, F. Bouchut, J. P. Vilotte, E. Lajeunesse, A. Aubertin, and M. Pirulli, "On the use of Saint Venant equations to simulate the spreading of a granular mass," J. Geophys. Res. 110, B09103, doi:10.1029/2004JB003161 (2005).

${ }^{19}$ E. Larrieu, L. Staron, and E. Hinch, "Raining into shallow water as a description of the collapse of a column of grains," J. Fluid Mech. 554, 259 (2006).

${ }^{20}$ O. Roche, M. A. Gilbertson, J. C. Phillips, and R. S. J. Sparks, "Experimental study of gas-fluidized granular flows with implications for pyroclastic flow emplacement," J. Geophys. Res. 109, B10201, doi:10.1029/2003JB002916 (2004).

${ }^{21}$ E. Meiburg and B. Kneller, "Turbidity currents and their deposits," Ann. Rev. Fluid Mech. 42, 135 (2010).

${ }^{22}$ E. Thompson and H. Huppert, "Granular column collapses: Further experimental results," J. Fluid Mech. 575, 177 (2007).

${ }^{23}$ F. Bonnet, T. Richard, and P. Philippe, "Sensitivity to solid volume fraction of gravitational instability in a granular medium," Granular Matter 12, 317 (2010).

${ }^{24}$ R. M. Iverson, M. E. Reid, N. R. Iverson, R. G. LaHusen, M. Logan, J. E. Mann, and D. L. Brien, "Acute sensitivity of landslide rates to initial soil porosity," Science 290, 513 (2000).

${ }^{25}$ D. G. Schaeffer and R. M. Iverson, "Steady and intermittent slipping in a model of landslide motion regulated by pore-pressure feedback," SIAM J. Appl. Math 69, 769 (2008).

${ }^{26}$ M. Pailha, M. Nicolas, and O. Pouliquen, "Initiation of underwater granular avalanches: Influence of the initial volume fraction," Phys. Fluids 20, 111701 (2008).

${ }^{27}$ M. Pailha and O. Pouliquen, "A two-phase flow description of the initiation of underwater granular avalanches," J. Fluid Mech. 633, 115 (2009).

${ }^{28}$ E. B. Pitman and L. Le, "A two-fluid model for avalanche and debris flows,” Philos. Trans. R. Soc. A 363, 1573 (2005).

${ }^{29}$ G. Y. Onoda and E. G. Liniger, "Random loose packings of uniform spheres and the dilatancy onset," Phys. Rev. Lett. 64, 2727 (1990).

${ }^{30} \mathrm{P}$. Philippe and D. Bideau, "Compaction dynamics of a granular medium under vertical tapping," Europhys. Lett. 60, 677 (2002).

${ }^{31}$ A. Daerr and S. Douady, "Sensitivity of granular surface flows to preparation,” Europhy. Lett. 47, 324 (1999). 